\title{
A LMI Approach to Stability Analysis and Synthesis of Impulsive Switched Systems with Time Delays *
}

\author{
Honglei $\mathrm{Xu}^{\mathrm{a}, \mathrm{c}, *}$ Xinzhi Liu ${ }^{\mathrm{b}}$ Kok Lay Teo ${ }^{\mathrm{c}}$

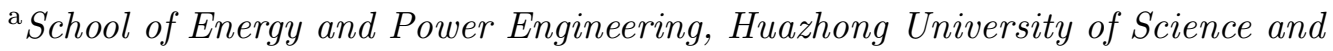 \\ Technology, Wuhan 430074, China \\ ${ }^{\mathrm{b}}$ Department of Applied Mathematics, University of Waterloo, Waterloo, Ontario \\ N2L 3G1, Canada \\ ${ }^{\mathrm{c}}$ Department of Mathematics and Statistics, Curtin University of Technology, \\ Perth, WA 6845, Australia
}

\begin{abstract}
This paper studies the asymptotic stability problem for a class of impulsive switched systems with time invariant delays based on linear matrix inequality(LMI) approach. Some sufficient conditions, which are independent of time delays and impulsive switching intervals, for ensuring asymptotical stability of these systems are derived by using a Lyapunov-Krasovskii technique. Moreover, some appropriate feedback controllers, which can stabilize the closed-loop systems, are constructed. Illustrative examples are presented to show the effectiveness of the results obtained.
\end{abstract}

Key words: Linear matrix inequallity, Impulsive switched system, Time delay, Asymptotic stability

$P A C S$ :

\section{Introduction}

Studies on dynamic systems with impulsive effects and switchings have arisen in various disciplines of science and engineering in recent years [1]-[5]. These

‡ This work was supported by the ARC under Grant DP0665948, the HUST Postdoctoral Foundation under Grant 200639 and the NSERC-Canada.

* Corresponding author. Tel.: +86 2787543630.

Email address: hongl_xu@163.com (Honglei Xu). 
systems are usually called impulsive switched systems. They present an effective and a convenient way to model those physical phenomena which exhibit abrupt changes at certain time points due to impulsive inputs or switchings. For these impulsive switched systems, there is an increasing interest among the control community in terms of stability analysis and the design of stabilizing feedback controllers so as to achieve a required stability performance. For example, in [1], some sufficient conditions for asymptotic stability of linear impulsive switched systems are obtained and the Lyapunov direct method is used to design linear feedback controllers which can robustly stabilize impulsive switched systems. In [2], a unified approach, which only requires a non increasing Lyapunov function along each part or unit of a system, is developed for analyzing the stability of impulsive switched and hybrid systems. For stability with definite attenuance, robust $H_{\infty}$ stability and stabilization with definite attenuance for impulsive switched systems with time-varying uncertainty are studied by using the LMI method in [3]. In addition, a procedure is also presented in [3] for the construction of a robust $H_{\infty}$ static state feedback controller that guarantees both robust stability with definite attenuance and robust $H_{\infty}$ performance.

On the other hand, time delay systems have also received an increasing attention among the control community. See, for example [6]-[9] and the references therein. The Lyapunov-Krasovskii Functional technique [6] is a useful technique, which extends the Lyapunov stability theory to time delay systems. Most of the results obtained for time delay systems are based on either Riccati inequalities or linear matrix inequalities. In this paper, our aim is to apply the Lyapunov-Krasovskii technique to impulsive delayed switched systems, to derive sufficient conditions for stability performance and to design feedback controllers based on the linear matrix inequalities approach. Our results are independent of time delays and impulsive and switching intervals. Some stability criteria expressed in terms of linear matrix inequalities are presented. They are easy to solve using the LMI toolbox within the Matlab environment.

The rest of the paper is organized as follows. In Section 2, an uncertain impulsive switched system with time delay is introduced. In Section 3, stability results based on the Lyapunov-Krasovskii method are obtained and some stability criteria expressed in terms of LMIs are derived. In Section 4, numerical examples are solved so as to illustrate the results obtained. Finally, we draw some concluding remarks in Section 5. 


\section{System description}

Consider the following impulsive switched system with time delay

$$
\begin{cases}\dot{x}(t)=\hat{A}_{i_{k}} x(t)+\hat{B}_{i_{k}} x(t-h)+C_{i_{k}} u(t), & t \neq t_{k} \\ \Delta x(t)=I_{k}(t, x)=D_{k} x(t), & t=t_{k} \\ x(t)=\varphi(t), & -\tau \leq t \leq 0 \\ \hat{A}_{i_{k}}=A_{i_{k}}+\Delta A_{i_{k}}, \hat{B}_{i_{k}}=B_{i_{k}}+\Delta B_{i_{k}}, & \end{cases}
$$

where $x(t) \in R^{n}$ and $u(t) \in R^{p}$, with $n, p \in N$, are the state and control vectors, respectively. $A_{i_{k}}, B_{i_{k}}, C_{i_{k}}$ and $D_{k}$ are constant real matrices of appropriate dimensions. $\Delta x(t)=x\left(t^{+}\right)-x\left(t^{-}\right), x\left(t^{-}\right)=\lim _{v \rightarrow 0+} x(t-v)$, $x\left(t^{+}\right)=\lim _{v \rightarrow 0+} x(t+v) . \lim _{v \rightarrow 0+} x\left(t_{k}-v\right)=x\left(t_{k}^{-}\right)=x\left(t_{k}\right)$ means that the solution of the impulsive switched system (1) is left continuous. $i_{k} \in\{1,2, \ldots m\}, k \in$ $N, m \in N$, is a discrete state variable, $t_{k}$ is an impulsive switching time point and $t_{0}<t_{1}<t_{2}<\ldots<t_{k}<\ldots<t_{\infty}$. Under the switching law of system (1), at the time point $t_{k}$, the system switches to the $i_{k}$ subsystem from the $i_{k-1}$ subsystem. Matrices $\Delta A_{i_{k}}(\cdot)$ and $\Delta B_{i_{k}}(\cdot)$ are unknown real norm-bounded matrix functions which represent time-varying parameter uncertainty. Assume that the admissible uncertainties are of the form

$$
\left[\Delta A_{i_{k}}(t) \Delta B_{i_{k}}(t)\right]=E_{i_{k}} F_{i_{k}}(t)\left[H_{i_{k}} J_{i_{k}}\right]
$$

and $E_{i_{k}}, H_{i_{k}}, J_{i_{k}}$ are known real constant matrices, $F_{i_{k}}(t)$ is an unknown real time-varying matrix satisfying $F_{i_{k}}^{T}(t) F_{i_{k}}(t)<I$, in which $I$ denotes the identity matrix of appropriate dimension.

\section{Asymptotic stability results}

In this section, stability criteria for impulsive switched systems with timeinvariant delays are derived. Before this, we need the following lemma.

Lemma 1 [3] Let $\bar{D}, \bar{E}$ and $\bar{\Delta}$ be real matrices of appropriate dimensions with $\bar{\Delta}^{T} \bar{\Delta} \leq I$. Then, for any scalar $\mu>0$, it holds that

$$
\bar{D} \bar{\Delta} \bar{E}+\bar{E}^{T} \bar{\Delta}^{T} \bar{D}^{T} \leq \frac{1}{\mu} \bar{D} \bar{D}^{T}+\mu \bar{E}^{T} \bar{E} .
$$


Our main results on the asymptotic stability of the impulsive switched systems with time-invariant delays are presented in the next two theorems.

Theorem 2 Suppose that there exist symmetric and positive definite matrices $P_{i_{k}}$ and $Q_{i_{k}}$, such that the following conditions are satisfied:

(a)

$$
\left[\begin{array}{cr}
\hat{A}_{i_{k}}^{T} P_{i_{k}}+P_{i_{k}} \hat{A}_{i_{k}}+Q_{i_{k}} & P_{i_{k}} \hat{B}_{i_{k}} \\
\hat{B}_{i_{k}}^{T} P_{i_{k}} & -Q_{i_{k}}
\end{array}\right]<0,
$$

where $\hat{A}_{i_{k}}=A_{i_{k}}+\Delta A_{i_{k}}, \hat{B}_{i_{k}}=B_{i_{k}}+\Delta B_{i_{k}}$, and

(b)

$$
\left[\begin{array}{cc}
P_{i_{k-1}} & \left(I+D_{k}\right)^{T} P_{i_{k}} \\
P_{i_{k}}\left(I+D_{k}\right) & P_{i_{k}}
\end{array}\right]>0 .
$$

Then, the trivial solution of the impulsive switched system (1) with the control input $u(t)=0$ is asymptotically stable.

Proof.

When $t \in\left(t_{k}, t_{k+1}\right]$, consider the Lyapunov-Krasovskii function candidate

$$
V(x(t))=x(t)^{T} P_{i_{k}} x(t)+\int_{t-h}^{t} x^{T}(s) Q_{i_{k}} x(s) d s .
$$

The derivative of the function defined by (6) along the solution of the impulsive switched system (1) is:

$$
\begin{gathered}
D^{+} V(x(t))=\dot{x}(t)^{T} P_{i_{k}} x(t)+x(t)^{T} P_{i_{k}} \dot{x}(t)+x(t)^{T} Q_{i_{k}} x(t) \\
-x^{T}(t-h) Q_{i_{k}} x(t-h) \\
=x^{T}(t)\left(\left(A_{i_{k}}+\Delta A_{i_{k}}\right)^{T} P_{i_{k}}+P\left(A_{i_{k}}+\Delta A_{i_{k}}\right)+Q_{i_{k}}\right) x(t) \\
+2 x^{T}(t-h)\left(B_{i_{k}}+\Delta B_{i_{k}}\right)^{T} P_{i_{k}} x(t) \\
-x^{T}(t-d) Q_{i_{k}} x(t-d) .
\end{gathered}
$$


(7) can be rewritten as:

$$
D^{+} V(x(t))=\left[\begin{array}{c}
x(t) \\
x(t-h)
\end{array}\right]^{T} \Theta_{i_{k}}\left[\begin{array}{c}
x(t) \\
x(t-h)
\end{array}\right]
$$

where $\Theta_{i_{k}}=\left[\begin{array}{cc}\hat{A}_{i_{k}}^{T} P_{i_{k}}+P_{i_{k}} \hat{A}_{i_{k}}+Q_{i_{k}} P_{i_{k}} \hat{B}_{i_{k}} \\ \hat{B}_{i_{k}}^{T} P_{i_{k}} & -Q_{i_{k}}\end{array}\right]$, where $\hat{A}_{i_{k}}=A_{i_{k}}+\Delta A_{i_{k}}, \hat{B}_{i_{k}}=$ $B_{i_{k}}+\Delta B_{i_{k}}$.

If (4) is satisfied, then

$$
D^{+} V(x(t))<0
$$

It means that the impulsive switched system is asymptotically stable, except possibly at the impulsive and switching points. Now, let us look at these time points. Note that at the time point $t_{k}, k=1,2, \ldots$, the system switches from the $i_{k-1}$ subsystem to the $i_{k}$ subsystem. To ensure the asymptotic stability, the following condition is required to be satisfied:

$$
\begin{gathered}
V\left(t_{k}^{+}\right)-V\left(t_{k}\right)=x\left(t_{k}^{+}\right)^{T} P_{i_{k}} x\left(t_{k}^{+}\right)-x\left(t_{k}\right)^{T} P_{i_{k-1}} x\left(t_{k}\right) \\
\quad \leq x\left(t_{k}\right)\left[\left(I+D_{k}\right)^{T} P_{i_{k}}\left(I+D_{k}\right)-P_{i_{k-1}}\right] x\left(t_{k}\right)<0 .
\end{gathered}
$$

This means that

$$
\left(I+D_{k}\right)^{T} P_{i_{k}}\left(I+D_{k}\right)-P_{i_{k-1}}<0
$$

or, equivalently,

$$
P_{i_{k-1}}-\left(I+D_{k}\right)^{T} P_{i_{k}}\left(I+D_{k}\right)>0
$$

From Schur complements [10], we see that the inequality (10) is equivalent to that of (5). This completes the proof.

Theorem 3 Suppose that there exist symmetric and positive definite matrices $P_{i_{k}}, Q_{i_{k}}$ and some positive scalars $\varepsilon_{1}, \varepsilon_{2}$, such that the following LMIs are satisfied: 
(a)

$$
\left[\begin{array}{ccc}
-Q_{i_{k}} & Q_{i_{k}} & 0 \\
Q_{i_{k}} & \Psi_{i_{k}} & P_{i_{k}} E_{i_{k}} \\
0 & E_{i_{k}}^{T} P_{i_{k}} & -\left(\varepsilon_{1}+\varepsilon_{2}\right)^{-1} I
\end{array}\right]<0
$$

where $\Psi_{i_{k}}=P_{i_{k}} A_{i_{k}}+A_{i_{k}}^{T} P_{i_{k}}+I+\varepsilon_{2}^{-1} H_{i_{k}}^{T} H_{i_{k}}$,

(b)

$$
\left[\begin{array}{cc}
-I & P_{i_{k}} B_{i_{k}} \\
B_{i_{k}}^{T} P_{i_{k}} & \varepsilon_{1}^{-1} J_{i_{k}}^{T} J_{i_{k}}-Q_{i_{k}}
\end{array}\right]<0
$$

(c)

$$
\left[\begin{array}{cc}
P_{i_{k-1}} & \left(I+D_{k}\right)^{T} P_{i_{k}} \\
P_{i_{k}}\left(I+D_{k}\right) & P_{i_{k}}
\end{array}\right]>0 .
$$

Then, the trivial solution of the impulsive switched system (1) with the control input $u(t)=0$ is asymptotically stable.

Proof.

Define

$$
Y_{i_{k}}=\left[\begin{array}{cc}
\hat{A}_{i_{k}}^{T} P_{i_{k}}+P_{i_{k}} \hat{A}_{i_{k}}+Q_{i_{k}} & P_{i_{k}} \hat{B}_{i_{k}} \\
\hat{B}_{i_{k}}^{T} P_{i_{k}} & -Q_{i_{k}}
\end{array}\right],
$$

where $\hat{A}_{i_{k}}=A_{i_{k}}+\Delta A_{i_{k}}, \hat{B}_{i_{k}}=B_{i_{k}}+\Delta B_{i_{k}}$, and

$$
S_{i_{k}}=\left[\begin{array}{cc}
A_{i_{k}}^{T} P_{i_{k}}+P_{i_{k}} A_{i_{k}}+Q_{i_{k}} & P_{i_{k}} B_{i_{k}} \\
B_{i_{k}}^{T} P_{i_{k}} & -Q_{i_{k}}
\end{array}\right] .
$$

Then, we have

$$
Y_{i_{k}}=S_{i_{k}}+\left[\begin{array}{cc}
\Delta A_{i_{k}}^{T} P_{i_{k}}+P_{i_{k}} \Delta A_{i_{k}} & P_{i_{k}} \Delta B_{i_{k}} \\
\Delta B_{i_{k}}^{T} P_{i_{k}} & 0
\end{array}\right]
$$




$$
\begin{aligned}
& =S_{i_{k}}+\left[\begin{array}{cc}
0 & P_{i_{k}} \Delta B_{i_{k}} \\
\Delta B_{i_{k}}^{T} P_{i_{k}} & 0
\end{array}\right] \\
& +\left[\begin{array}{cc}
\Delta A_{i_{k}}^{T} P_{i_{k}}+P_{i_{k}} \Delta A_{i_{k}} & 0 \\
0 & 0
\end{array}\right] \\
& =S_{i_{k}}+\left[\begin{array}{cc}
0 & P_{i_{k}} E_{i_{k}} F_{i_{k}}(t) J_{i_{k}} \\
J_{i_{k}}^{T} F_{i_{k}}^{T}(t) E_{i_{k}}^{T} P_{i_{k}} & 0
\end{array}\right] \\
& +\left[\begin{array}{cc}
H_{i_{k}}^{T} F_{i_{k}}^{T}(t) E_{i_{k}}^{T} P_{i_{k}}+P_{i_{k}} E_{i_{k}} F_{i_{k}}(t) H_{i_{k}} & 0 \\
0 & 0
\end{array}\right] \\
& =S_{i_{k}}+\left[\begin{array}{c}
P_{i_{k}} E_{i_{k}} \\
0
\end{array}\right]\left[F_{i_{k}}(t)\right]\left[\begin{array}{ll}
0 & J_{i_{k}}
\end{array}\right] \\
& +\left[\begin{array}{c}
0 \\
J_{i_{k}}^{T}
\end{array}\right]\left[F_{i_{k}}^{T}(t)\right]\left[E_{i_{k}}^{T} P_{i_{k}} 0\right] \\
& +\left[\begin{array}{cc}
H_{i_{k}}^{T} F_{i_{k}}^{T}(t) E_{i_{k}}^{T} P_{i_{k}}+P_{i_{k}} E_{i_{k}} F_{i_{k}}(t) H_{i_{k}} & 0 \\
0 & 0
\end{array}\right] \\
& \leq S_{i_{k}}+\varepsilon_{1}\left[\begin{array}{cc}
P_{i_{k}} E_{i_{k}} E_{i_{k}}^{T} P_{i_{k}} & 0 \\
0 & 0
\end{array}\right]+\varepsilon_{1}^{-1}\left[\begin{array}{cc}
0 & 0 \\
0 & J_{i_{k}}^{T} J_{i_{k}}
\end{array}\right] \\
& +\left[\begin{array}{cc}
\varepsilon_{2} P_{i_{k}} E_{i_{k}} E_{i_{k}}^{T} P_{i_{k}}+\varepsilon_{2}^{-1} H_{i_{k}}^{T} H_{i_{k}} & 0 \\
0 & 0
\end{array}\right] \\
& =\left[\begin{array}{cc}
A_{i_{k}}^{T} P_{i_{k}}+P_{i_{k}} A_{i_{k}}+Q_{i_{k}} & P_{i_{k}} B_{i_{k}} \\
B_{i_{k}}^{T} P_{i_{k}} & \varepsilon_{1}^{-1} J_{i_{k}}^{T} J_{i_{k}}-Q_{i_{k}}
\end{array}\right] \\
& +\left[\begin{array}{cc}
\left(\varepsilon_{1}+\varepsilon_{2}\right) P_{i_{k}} E_{i_{k}} E_{i_{k}}^{T} P_{i_{k}}+\varepsilon_{2}^{-1} H_{i_{k}}^{T} H_{i_{k}} & 0 \\
0 & 0
\end{array}\right] \\
& =\left[\begin{array}{cc}
-I & P_{i_{k}} B_{i_{k}} \\
B_{i_{k}}^{T} P_{i_{k}} & \varepsilon_{1}^{-1} J_{i_{k}}^{T} J_{i_{k}}-Q_{i_{k}}
\end{array}\right]
\end{aligned}
$$




$$
+\left[\begin{array}{cc}
Z_{i_{k}} & 0 \\
0 & 0
\end{array}\right]
$$

where

$$
\begin{gathered}
Z_{i_{k}}=A_{i_{k}}^{T} P_{i_{k}}+P_{i_{k}} A_{i_{k}}+Q_{i_{k}}+\left(\varepsilon_{1}+\varepsilon_{2}\right) P_{i_{k}} E_{i_{k}} E_{i_{k}}^{T} P_{i_{k}}+I \\
+\varepsilon_{2}^{-1} H_{i_{k}}^{T} H_{i_{k}} .
\end{gathered}
$$

The stability condition $D^{+} V(x(t))<0$ can be obtained if the inequalities

$$
\left[\begin{array}{cc}
-I & P_{i_{k}} B_{i_{k}} \\
B_{i_{k}}^{T} P_{i_{k}} & \varepsilon_{1}^{-1} J_{i_{k}}^{T} J_{i_{k}}-Q_{i_{k}}
\end{array}\right]<0,
$$

and

$$
Z_{i_{k}}<0
$$

hold.

Then, (18) will hold if the following condition is satisfied:

$$
\left[\begin{array}{ccc}
-I & 0 & 0 \\
0 & Z_{i_{k}} & 0 \\
0 & 0 & -I
\end{array}\right]<0
$$

Define

$$
W_{i_{k}}=\left[\begin{array}{ccc}
Q_{i_{k}}^{1 / 2} & 0 & 0 \\
-Q_{i_{k}}^{1 / 2} & I & -\left(\varepsilon_{1}+\varepsilon_{2}\right)^{\frac{1}{2}} P_{i_{k}} E_{i_{k}} \\
0 & 0 & \left(\varepsilon_{1}+\varepsilon_{2}\right)^{-\frac{1}{2}} I
\end{array}\right] .
$$

Then, by left multiplying and right multiplying (19) by $W_{i_{k}}$ and $W_{i_{k}}^{T}$, we obtain

$$
\left[\begin{array}{ccc}
-Q_{i_{k}} & Q_{i_{k}} & 0 \\
Q_{i_{k}} & \Psi_{i_{k}} & P_{i_{k}} E_{i_{k}} \\
0 & E_{i_{k}}^{T} P_{i_{k}} & -\left(\varepsilon_{1}+\varepsilon_{2}\right)^{-1} I
\end{array}\right]<0,
$$


where $\Psi_{i_{k}}=P_{i_{k}} A_{i_{k}}+A_{i_{k}}^{T} P_{i_{k}}+I+\varepsilon_{2}^{-1} H_{i_{k}}^{T} H_{i_{k}}$.

This completes the proof.

\section{Design of feedback controllers}

In this section, we focus on the design of feedback controllers of the form $u(t)=L_{i_{k}} x(t)$ for impulsive switched systems with time delays. Thus, the objective of this section is to provide a computational procedure to construct an appropriate $L_{i_{k}}$ such that the corresponding closed-loop system is stable.

Theorem 4 Suppose that there exist symmetric and positive definite matrices $P_{i_{k}}$ and $Q_{i_{k}}$, such that the following conditions are satisfied:

(a)

$$
\left[\begin{array}{cc}
\bar{A}_{i_{k}}^{T} P_{i_{k}}+P_{i_{k}} \bar{A}_{i_{k}}+Q_{i_{k}} & P_{i_{k}} \hat{B}_{i_{k}} \\
\hat{B}_{i_{k}}^{T} P_{i_{k}} & -Q_{i_{k}}
\end{array}\right]<0,
$$

where $\bar{A}_{i_{k}}=A_{i_{k}}+\Delta A_{i_{k}}+C_{i_{k}} L_{i_{k}}, \hat{B}_{i_{k}}=B_{i_{k}}+\Delta B_{i_{k}}$, and

(b)

$$
\left[\begin{array}{cc}
P_{i_{k-1}} & \left(I+D_{k}\right)^{T} P_{i_{k}} \\
P_{i_{k}}\left(I+D_{k}\right) & P_{i_{k}}
\end{array}\right]>0 .
$$

Then, the trivial solution of the impulsive switched system (1) is asymptotically stable.

Proof.

Substitute $u(t)=L_{i_{k}} x(t)$ in equation (1). Then, the corresponding impulsive switched system (1) become

$$
\left\{\begin{array}{ll}
\dot{x}(t)=\bar{A}_{i_{k}} x(t)+\hat{B}_{i_{k}} x(t-h) & t \neq t_{k} \\
\Delta x(t)=I_{k}(t, x)=D_{k} x(t) & t=t_{k} \\
x(t)=\varphi(t) & -\tau \leq t \leq 0
\end{array},\right.
$$

where $\bar{A}_{i_{k}}=A_{i_{k}}+\Delta A_{i_{k}}+C_{i_{k}} L_{i_{k}}, \hat{B}_{i_{k}}=B_{i_{k}}+\Delta B_{i_{k}}$. 
Hence, the conclusion of the theorem follows readily from the proof of Theorem 2. This completes the proof.

Theorem 5 Support that there exist symmetric and positive definite matrices $P_{i_{k}}, Q_{i_{k}}$ and some positive scalars $\varepsilon_{1}, \varepsilon_{2}$, such that the following matrix inequalities are satisfied:

(a)

$$
\left[\begin{array}{ccc}
-Q_{i_{k}} & Q_{i_{k}} & 0 \\
Q_{i_{k}} & \bar{\Psi}_{i_{k}} & P_{i_{k}} E_{i_{k}} \\
0 & E_{i_{k}}^{T} P_{i_{k}} & -\left(\varepsilon_{1}+\varepsilon_{2}\right)^{-1} I
\end{array}\right]<0
$$

where $\bar{\Psi}_{i_{k}}=P_{i_{k}} A_{i_{k}}+A_{i_{k}}^{T} P_{i_{k}}+I+\varepsilon_{2}^{-1} H_{i_{k}}^{T} H_{i_{k}}-P_{i_{k}} C_{i_{k}} C_{i_{k}}^{T} P_{i_{k}}$,

(b)

$$
\left[\begin{array}{cc}
-I & P_{i_{k}} B_{i_{k}} \\
B_{i_{k}}^{T} P_{i_{k}} & \varepsilon_{1}^{-1} J_{i_{k}}^{T} J_{i_{k}}-\varepsilon_{1} \varepsilon_{2} Q_{i_{k}}
\end{array}\right]<0
$$

(c)

$$
\left[\begin{array}{cc}
P_{i_{k-1}} & \left(I+D_{k}\right)^{T} P_{i_{k}} \\
P_{i_{k}}\left(I+D_{k}\right) & P_{i_{k}}
\end{array}\right]>0 .
$$

Then, the trivial solution of impulsive switched system (1) is asymptotically stable. Moreover,

$$
u(t)=L_{i_{k}} x(t), \quad L_{i_{k}}=-\frac{1}{2} C_{i_{k}}^{T} P_{i_{k}}
$$

is a feedback controller which stabilizes the corresponding closed-loop impulsive switched system.

Proof: 
Substitute the feedback controllers $u(t)=L_{i_{k}} x(t)$, where $L_{i_{k}}=-\frac{1}{2} C_{i_{k}}^{T} P_{i_{k}}$, in (1). Then, the corresponding impulsive switched system (1) becomes

$$
\begin{cases}\dot{x}(t)=\tilde{A}_{i_{k}} x(t)+\bar{B}_{i_{k}} x(t-h), & t \neq t_{k} \\ \Delta x(t)=I_{k}(t, x)=D_{k} x(t), & t=t_{k} \\ x(t)=\varphi(t), & -\tau \leq t \leq 0\end{cases}
$$

where $\tilde{A}_{i_{k}}=A_{i_{k}}-\frac{1}{2} C_{i_{k}} C_{i_{k}}^{T} P_{i_{k}}+E_{i_{k}} F_{i_{k}} H_{i_{k}}, \bar{B}_{i_{k}}=B_{i_{k}}+E_{i_{k}} F_{i_{k}} J_{i_{k}}$. By Theorem 3 , it is easy to show that the closed-loop impulsive switched system (29) is asymptotically stable. This completes the proof.

Theorem 6 Suppose that there exist symmetric and positive definite matrices $P_{i_{k}}, Q_{i_{k}}$ and some positive scalars $\varepsilon_{1}, \varepsilon_{2}$, such that the following LMIs are satisfied: (a)

$$
\left[\begin{array}{ccc}
-Q_{i_{k}} & Q_{i_{k}} & 0 \\
Q_{i_{k}} & \Psi_{i_{k}} & P_{i_{k}} U_{i_{k}} \\
0 & U_{i_{k}}^{T} P_{i_{k}} & -I
\end{array}\right]<0
$$

where $U_{i_{k}} U_{i_{k}}^{T}=\left(\varepsilon_{1}+\varepsilon_{2}\right) E_{i_{k}} E_{i_{k}}^{T}-C_{i_{k}} C_{i_{k}}^{T}, \Psi_{i_{k}}=P_{i_{k}} A_{i_{k}}+A_{i_{k}}^{T} P_{i_{k}}+I+\varepsilon_{2}^{-1} H_{i_{k}}^{T} H_{i_{k}}$, (b)

$$
\left[\begin{array}{cc}
-I & P_{i_{k}} B_{i_{k}} \\
B_{i_{k}}^{T} P_{i_{k}} & \varepsilon_{1}^{-1} J_{i_{k}}^{T} J_{i_{k}}-Q_{i_{k}}
\end{array}\right]<0,
$$

(c)

$$
\left[\begin{array}{cc}
P_{i_{k-1}} & \left(I+D_{k}\right)^{T} P_{i_{k}} \\
P_{i_{k}}\left(I+D_{k}\right) & P_{i_{k}}
\end{array}\right]>0 .
$$

Then, the trivial solution of the impulsive switched system (1) is asymptotically stable. Moreover,

$$
u(t)=L_{i_{k}} x(t), \quad L_{i_{k}}=-\frac{1}{2} C_{i_{k}}^{T} P_{i_{k}}
$$

is a feedback controller which stabilizes the corresponding closed-loop impulsive switched systems. 
Proof.

The matrix inequality (25) can be written as

$$
\begin{gathered}
A_{i_{k}}^{T} P_{i_{k}}+P_{i_{k}} A_{i_{k}}+Q_{i_{k}} \\
+P_{i_{k}} U_{i_{k}}^{T} U_{i_{k}} P_{i_{k}}+I+\varepsilon_{2}^{-1} H_{i_{k}}^{T} H_{i_{k}}<0
\end{gathered}
$$

Let

$$
\begin{gathered}
\hat{Z}_{i_{k}}=A_{i_{k}}^{T} P_{i_{k}}+P_{i_{k}} A_{i_{k}}+Q_{i_{k}} \\
+P_{i_{k}} U_{i_{k}}^{T} U_{i_{k}} P_{i_{k}}+I+\varepsilon_{2}^{-1} H_{i_{k}}^{T} H_{i_{k}} .
\end{gathered}
$$

Then, we can see that (34) is equivalent to the following condition

$$
\left[\begin{array}{ccc}
-I & 0 & 0 \\
0 & \hat{Z}_{i_{k}} & 0 \\
0 & 0 & -I
\end{array}\right]<0
$$

Define

$$
\hat{W}_{i_{k}}=\left[\begin{array}{ccc}
Q_{i_{k}}^{1 / 2} & 0 & 0 \\
-Q_{i_{k}}^{1 / 2} & I & -P_{i_{k}} U_{i_{k}} \\
0 & 0 & I
\end{array}\right] .
$$

Then, left multiplying and right multiplying (36) by $\hat{W}_{i_{k}}$ and $\hat{W}_{i_{k}}^{T}$ respectively, we obtain

$$
\left[\begin{array}{ccc}
-Q_{i_{k}} & Q_{i_{k}} & 0 \\
Q_{i_{k}} & \Psi_{i_{k}} & P_{i_{k}} U_{i_{k}} \\
0 & U_{i_{k}}^{T} P_{i_{k}} & -I
\end{array}\right]<0
$$

where $\Psi_{i_{k}}=P_{i_{k}} A_{i_{k}}+A_{i_{k}}^{T} P_{i_{k}}+I+\varepsilon_{2}^{-1} H_{i_{k}}^{T} H_{i_{k}}$.

The rest of the theorem can be obtained readily from Theorem 5 . This completes the proof.

Remark 7 Theorem 6 offers a LMI based design of a linear memoryless state 
feedback controller, which will robustly stabilize the corresponding controlled impulsive delayed switched system.

Remark 8 Inequalities (30)-(32) of Theorem 6 are expressed in terms of some linear matrix inequalities with variables $\varepsilon_{1}, \varepsilon_{2}, Q_{i_{k}}, P_{i_{k}}$. The feasibility of these LMIs can be solved by using feasp command in the LMI toolbox within the MATLAB environment. Once a feasible solution of these LMIs is found, the required solution can obtained readily.

We now consider the case of no switchings in the system (1). For this case, system (1) becomes:

$$
\begin{cases}\dot{x}(t)=\hat{A} x(t)+\hat{B} x(t-h)+C u(t), & t \neq t_{k} \\ \Delta x(t)=I_{k}(t, x)=D_{k} x(t), & t=t_{k} \\ x(t)=\varphi(t), & -\tau \leq t \leq 0\end{cases}
$$

where $\hat{A}=A+\Delta A, \hat{B}=B+\Delta B$.

The admissible uncertainties are of the form

$$
[\Delta A(t) \Delta B(t)]=E F[H J]
$$

where $E, H, J$ are known real constant matrices, $F(t)$ is an unknown real time-varying matrix satisfying $F^{T}(t) F(t)<I$.

Corollary 9 Suppose that there exist symmetric and positive definite matrices $P, Q$ and some positive scalars $\varepsilon_{1}, \varepsilon_{2}$, such that the following LMIs are satisfied:

(a)

$$
\left[\begin{array}{ccc}
-Q & Q & 0 \\
Q & \Psi & P E \\
0 & E^{T} P-\left(\varepsilon_{1}+\varepsilon_{2}\right)^{-1} I
\end{array}\right]<0
$$

where $\Psi=P A+A^{T} P+I+\varepsilon_{2}^{-1} H^{T} H$,

(b)

$$
\left[\begin{array}{cc}
-I & P B \\
B^{T} P & \varepsilon_{1}^{-1} J^{T} J-Q
\end{array}\right]<0
$$


(c)

$$
\left[\begin{array}{cc}
P & \left(I+D_{k}\right)^{T} P \\
P\left(I+D_{k}\right) & P
\end{array}\right]>0 .
$$

Then, the trivial solution of the uncertain impulsive system (39) with the control input $u(t)=0$ is asymptotically stable.

Corollary 10 Suppose that there exist symmetric and positive definite matrices $P, Q$ and some positive scalars $\varepsilon_{1}, \varepsilon_{2}$, such that the following LMIs are satisfied:

(a)

$$
\left[\begin{array}{ccc}
-Q & Q & 0 \\
Q & P A+A^{T} P+I+\varepsilon_{2}^{-1} H^{T} H & P U \\
0 & U^{T} P & -I
\end{array}\right]<0
$$

where $U U^{T}=\left(\varepsilon_{1}+\varepsilon_{2}\right) E E^{T}-C C^{T}$,

(b)

$$
\left[\begin{array}{cc}
-I & P B \\
B^{T} P & \varepsilon_{1}^{-1} J^{T} J-Q
\end{array}\right]<0,
$$

(c)

$$
\left[\begin{array}{cc}
P & \left(I+D_{k}\right)^{T} P \\
P\left(I+D_{k}\right) & P
\end{array}\right]>0 .
$$

Then, the trivial solution of the uncertain impulsive system (39) is asymptotically stable. Moreover,

$$
u(t)=L x(t), \quad L=-\frac{1}{2} C^{T} P
$$

is a feedback controller which stabilizes the corresponding closed-loop impulsive systems. 


\section{$5 \quad$ Numerical examples and simulations}

We consider two examples in this section.

Example 1 Consider the following impulsive delayed switched system under a given switching law. That is, the switching status alternates as $i_{1} \rightarrow i_{2} \rightarrow$ $i_{1} \rightarrow i_{2} \rightarrow \ldots$, and $F_{1}=F_{2}=\sin (10 * t)$. We consider robust performance of the system using Theorem 3. The parameters of the system are specified as follows:

$$
\begin{gathered}
A_{1}=\left[\begin{array}{rr}
-3 & -0.8 \\
-0.8 & -2.8
\end{array}\right], E_{1}=\left[\begin{array}{ll}
0.5 & 0.4 \\
0.2 & 0.4
\end{array}\right], \\
H_{1}=\left[\begin{array}{ll}
0.2 & 0.4 \\
0.2 & 0.1
\end{array}\right], B_{1}=\left[\begin{array}{ll}
0.8 & 1 \\
1.5 & 1
\end{array}\right], \\
J_{1}=\left[\begin{array}{ll}
0.2 & 0.3 \\
0.2 & 0.1
\end{array}\right], A_{2}=\left[\begin{array}{ll}
-2.8 & -1 \\
-0.7 & -3.1
\end{array}\right], \\
E_{2}=\left[\begin{array}{ll}
0.3 & 0.1 \\
0.6 & 0.4
\end{array}\right], H_{2}=\left[\begin{array}{ll}
0.1 & 0.1 \\
0.5 & -0.1
\end{array}\right], \\
B_{2}=\left[\begin{array}{cc}
1.5 & -0.3 \\
-0.2 & 1
\end{array}\right], J_{2}=\left[\begin{array}{ll}
0.2 & 0.1 \\
0.3 & 0.2
\end{array}\right] .
\end{gathered}
$$

Choose $\varepsilon_{1}=\varepsilon_{2}=1$. Then, we use the feasp command within the MATLAB environment to calculate the positive define symmetric matrices, giving

$$
\begin{gathered}
P_{1}=\left[\begin{array}{cc}
5.5303 & -3.6741 \\
-3.6741 & 3.4226
\end{array}\right], P_{2}=\left[\begin{array}{ll}
1.4999 & 0.5335 \\
0.5335 & 2.2719
\end{array}\right], \\
Q_{1}=\left[\begin{array}{cc}
12.8403 & -7.4540 \\
-7.4540 & 7.2573
\end{array}\right], \quad Q_{2}=\left[\begin{array}{ll}
5.7463 & 1.7452 \\
1.7452 & 6.3872
\end{array}\right] .
\end{gathered}
$$

It means that there exist $P_{1}, Q_{1}, P_{2}, Q_{2}$ such that (11)-(13) are satisfied, so the system is asymptotically stable by Theorem 3 . Let $(2,-1.5)$ be the initial point. Fig. 1-Fig. 2 show the state trajectories of the impulsive delayed 


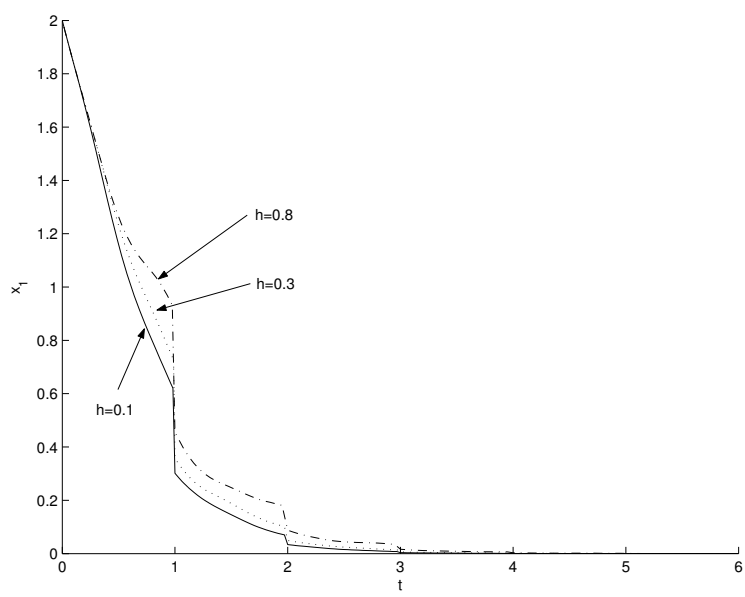

Fig. 1. State trajectories of $x_{1}$. The solid, dotted, and dashed-dotted curves are for cases with $h=0.1,0.3$ and 0.8 , respectively.

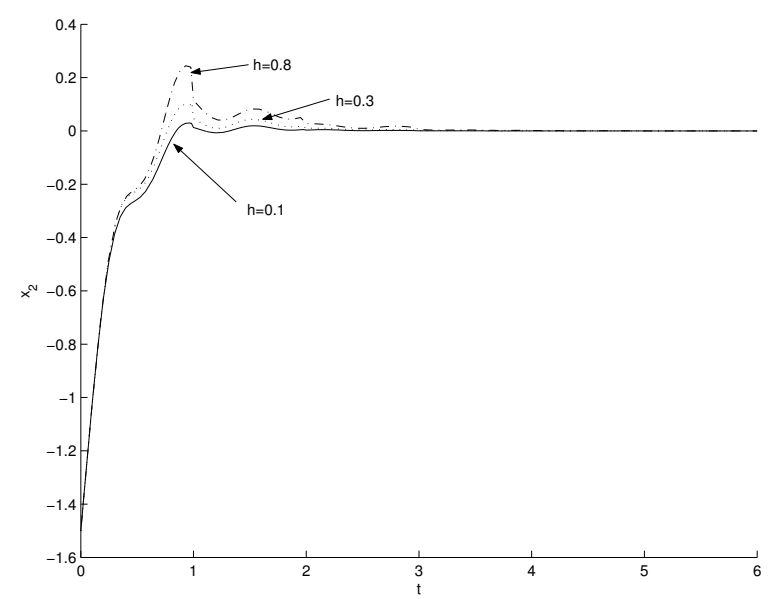

Fig. 2. State trajectories of $x_{2}$. The solid, dotted, and dashed-dotted curves are for cases with $h=0.1,0.3$ and 0.8 , respectively.

switched system with a constant interval time $\Delta t_{k} \equiv 1$. The solid, dotted, and dashed-dotted curves are for cases with the delay $h$ chosen as $0.1,0.3$, 0.8, respectively. Fig. 1-Fig. 2 also show that a longer time delay will result in a slower convergence rate. Fig. 3-Fig. 4 show the state trajectories of the impulsive delayed switched system with a constant time delay $h \equiv 0.2$. The solid, dotted, and dashed-dotted curves are for cases with the interval $\Delta t_{k}$ chosen as 0.5 and 1, 2, respectively. Fig. 3-Fig. 4 also show that a longer interval will result in a slower convergence rate.

Example 2 Consider another impulsive delayed switched system under the same switching law as in Example 5.1. The corresponding parameters are spec- 


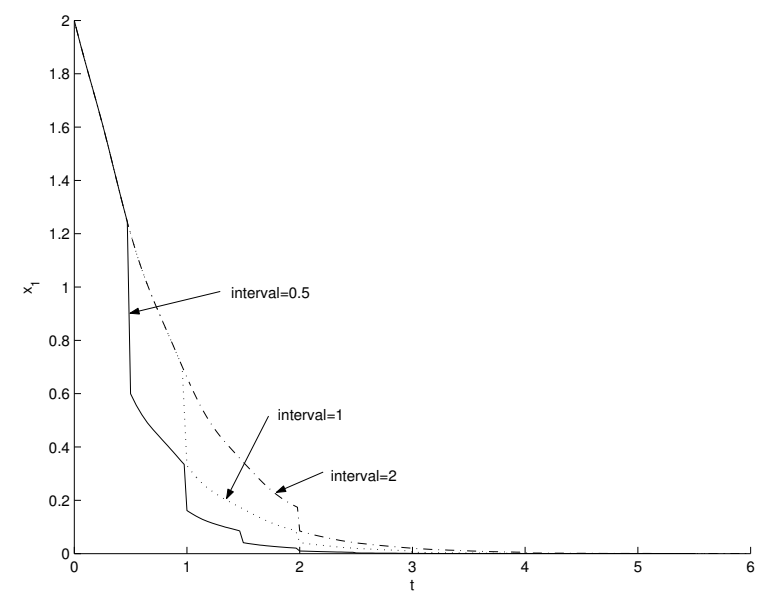

Fig. 3. State trajectories of $x_{1}$. The solid, dotted, and dashed-dotted curves are for cases with interval $=0.5,1$ and 2 , respectively.

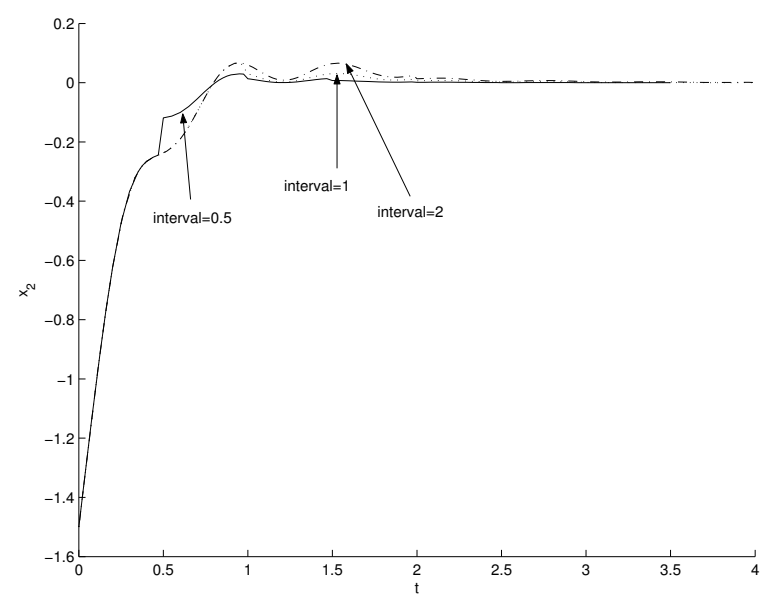

Fig. 4. State trajectories of $x_{2}$. The solid, dotted, and dashed-dotted curves are for cases with interval $=0.5,1$ and 2 , respectively.

ified as follows:

$$
\begin{gathered}
A_{1}=\left[\begin{array}{ll}
-3.3 & -1.1 \\
-0.6 & -3.2
\end{array}\right], \quad E_{1}=\left[\begin{array}{ll}
0.5 & 0.4 \\
0.2 & 0.4
\end{array}\right], \\
H_{1}=\left[\begin{array}{ll}
0.2 & 0.4 \\
0.2 & 0.1
\end{array}\right], B_{1}=\left[\begin{array}{ll}
0.8 & 1 \\
1.5 & 1
\end{array}\right], \\
J_{1}=\left[\begin{array}{ll}
0.2 & 0.3 \\
0.2 & 0.1
\end{array}\right], A_{2}=\left[\begin{array}{ll}
-2.5 & -1.2 \\
-0.8 & -2.5
\end{array}\right],
\end{gathered}
$$




$$
\begin{gathered}
E_{2}=\left[\begin{array}{ll}
0.3 & 0.1 \\
0.6 & 0.4
\end{array}\right], \quad H_{2}=\left[\begin{array}{cc}
0.1 & 0.1 \\
0.5 & -0.1
\end{array}\right], \\
B_{2}=\left[\begin{array}{cc}
1.5 & -0.3 \\
-0.2 & 1
\end{array}\right], \quad D_{1}=D_{2}=\left[\begin{array}{cc}
-0.5 & 0 \\
0 & -0.5
\end{array}\right], \\
J_{2}=\left[\begin{array}{ll}
0.2 & 0.1 \\
0.3 & 0.2
\end{array}\right], \quad C_{1}=\left[\begin{array}{ll}
0.6 & 0.4 \\
0.2 & 0.2
\end{array}\right], \quad C_{2}=\left[\begin{array}{ll}
0.2 & 0.2 \\
0.2 & 0.4
\end{array}\right] .
\end{gathered}
$$

Choose $\varepsilon_{1}=\varepsilon_{2}=1$. Then, using the feasp command within the MATLAB environment, we calculate the positive define symmetric matrices as given below.

$$
\begin{gathered}
P_{1}=\left[\begin{array}{cc}
0.8684 & -0.1629 \\
-0.1629 & 0.6567
\end{array}\right], P_{2}=\left[\begin{array}{cc}
0.9749 & 0.0588 \\
0.0588 & 0.9857
\end{array}\right], \\
Q_{1}=\left[\begin{array}{cc}
3.1070 & -0.7158 \\
-0.7158 & 1.9694
\end{array}\right], Q_{2}=\left[\begin{array}{cc}
2.4971 & -0.2988 \\
-0.2988 & 1.3960
\end{array}\right] .
\end{gathered}
$$

Furthermore, we also obtain the following linear memoryless time-variant controller

$$
\begin{aligned}
& u(t)=L_{i_{k}} x(t), \\
& L_{1}=\left[\begin{array}{ll}
-0.2442 & -0.0168 \\
-0.1574 & -0.0331
\end{array}\right], L_{2}=\left[\begin{array}{l}
-0.1034-0.1045 \\
-0.1093-0.2030
\end{array}\right] \text {. }
\end{aligned}
$$

Given initial point $(1,-1)$. Fig. 5 and Fig. 6 show the state trajectories of the impulsive delayed switched system with a constant interval time $\Delta t_{k} \equiv 1$. The solid, dotted, and dashed-dotted curves are for cases with the delay $h$ chosen as 0.1, 0.3, and 0.8, respectively. Fig. 7 and Fig. 8 show that the state trajectories of the impulsive delayed switched system with a constant time delay $h \equiv 0.2$. The solid, dotted, and dashed-dotted curves are for cases with the interval $\Delta t_{k}$ chosen as $0.5,1$, and 2, respectively. Fig. 5-Fig. 8 also show that with the linear feedback controller, the uncertain impulsive switched system converges to the equilibrium point. 


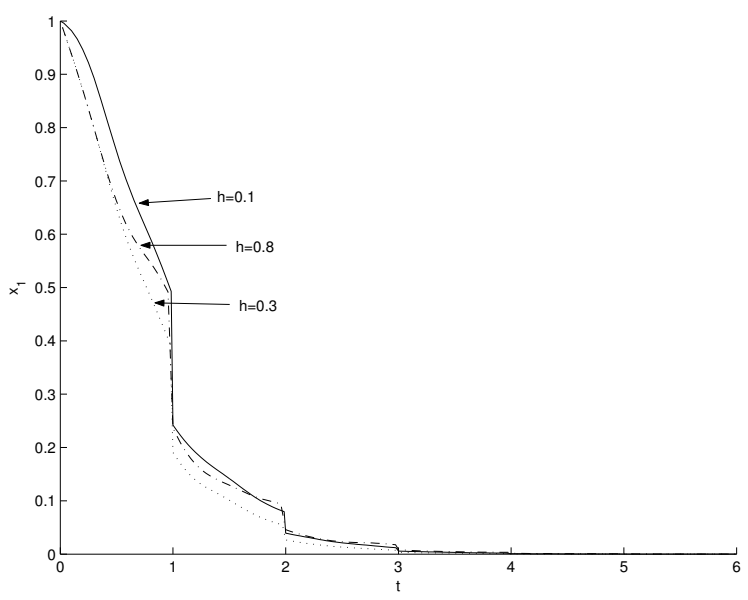

Fig. 5. State trajectories of $x_{1}$. The solid, dotted, and dashed-dotted curves are for cases with $h=0.1,0.3$ and 0.8 , respectively.

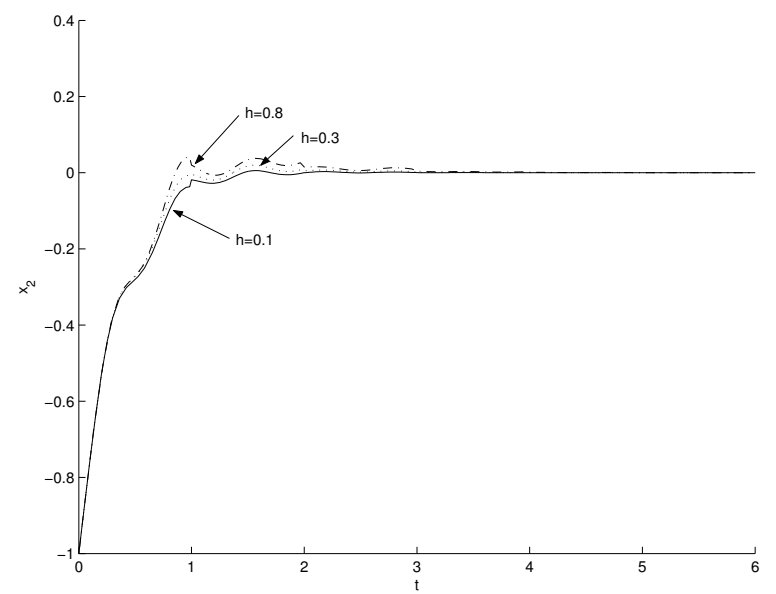

Fig. 6. State trajectories of $x_{2}$. The solid, dotted, and dashed-dotted curves are for cases with $h=0.1,0.3$ and 0.8 , respectively.

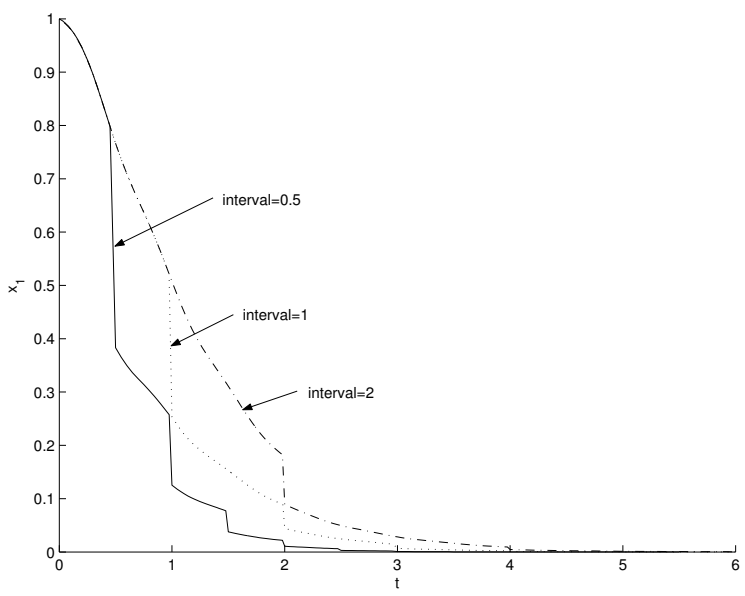

Fig. 7. State trajectories of $x_{1}$. The solid, dotted, and dashed-dotted curves are for cases with interval $=0.5,1$ and 2 , respectively. 


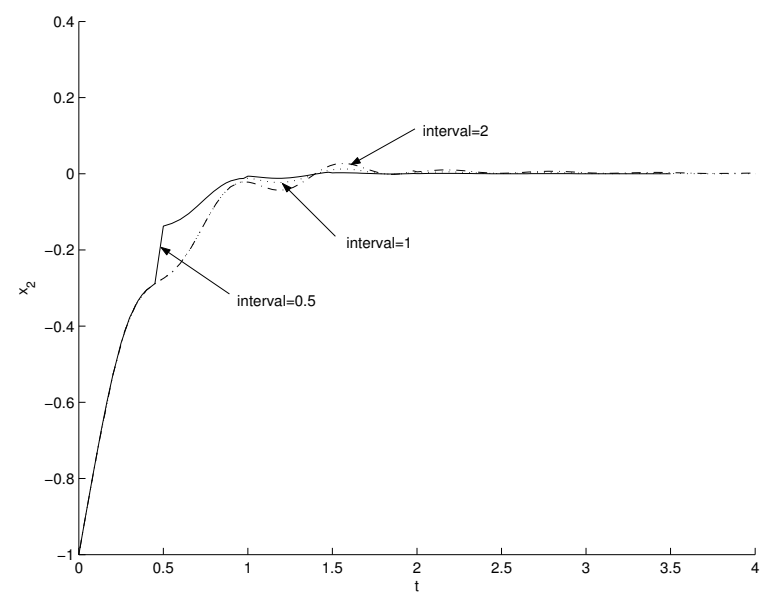

Fig. 8. State trajectories of $x_{2}$. The solid, dotted, and dashed-dotted curves are for cases with interval $=0.5,1$ and 2 , respectively.

\section{Conclusion}

The stability problem of a class of impulsive delayed switched systems was studied. By constructing appropriate Lyapunov-Krasovskii functions and using LMI approach, some asymptotic stability criteria were obtained and some appropriate feedback controllers were constructed. As an illustration, two numerical examples were solved using the results obtained in this paper.

\section{References}

[1] X. Ding and H. Xu, Robust stability and stabilization of a class of impulsive switched systems, Dynamics of Continuous Discrete and Impulsive SystemsSeries B, Sp. Iss. SI, 2(2005) 795-798.

[2] C. Y. Wen, Z. G. Li and Y. C. Soh, A unified approach for stability analysis of impulsive hybrid systems, Proceedings of the 38th IEEE Conference on Decision and Control, 5(1999) 4398-4403.

[3] H. Xu, X. Liu and K. L. Teo, Robust $H \infty$ stabilization with definite attendance of uncertain impulsive switched systems, Journal of ANZIAM 46(2005) 471-484.

[4] D. J. Hill, Z. Guan and X. Shen, On hybrid impulsive and switching systems and aplication to nonlinear control, IEEE Transactions on Automatic Control 50(2005) 1058-1062.

[5] X. Liu, B. Liu and X. Liao, Stability and robustness of quasi-linear impulsive hybrid systems, J. Math. Anal. Appl. 283(2003) 416-430.

[6] K. Gu, Survey on recent results in the stability and control of time delay systems, Journal of Dynamic Systems, Measurement and Control 125(2003) $158-165$. 
[7] X. Li and C. E. de Souza, Delay dependent robust stability and stabilization of uncertain time delay systems: a linear matrix inequality approach, IEEE Transaction of Automatic Control 42(1997) 1144-1148.

[8] J. C. Doyle, K. Zhou and K. Glover, Robust and Optimal Control, Prentice Hall, NJ, 1996.

[9] P. Park, A delay-dependent stability criterion for systems with uncertain timeinvariant delays, IEEE Transaction on Automatic Control 44(1999) 876-877.

[10] E. Feron S. Boyd, L. E. Ghaoui and V. Balakrishnan, Linear Matrix Inequalities in System and Control Theory, SIAM, Philadelphia, 1994.

[11] H.L. Xu, X.Z. Liu and K.L. Teo, Robust $\mathrm{H}_{\infty}$ stabilization with definite attendance of uncertain impulsive switched systems, Journal of ANZIAM 46(2005) 471-484.

[12] J. K. Hale and S. M. Verduyn Lunel, Introduction to Functional Differential Equations, Springer-Verlag, New York, 1993. 